\title{
Analyse multi-niveaux de l'activité en situation de formation : proposition méthodologique pour une étude synchronique et diachronique
}

Pluri-scalar analysis in training environment: a methodological proposition for a synchronic and diachronic study

Marlène Manach

\section{OpenEdition}

Journals

Édition électronique

URL : http://journals.openedition.org/ree/695

DOI : 10.4000/ree.695

ISSN : 1954-3077

Éditeur

Université de Nantes

Référence électronique

Marlène Manach, «Analyse multi-niveaux de l'activité en situation de formation : proposition méthodologique pour une étude synchronique et diachronique », Recherches en éducation [En ligne] 38 | 2019, mis en ligne le 01 novembre 2019, consulté le 03 septembre 2020. URL : http:// journals.openedition.org/ree/695; DOI : https://doi.org/10.4000/ree.695

\section{(c) (i) $\ominus$}

Recherches en éducation est mise à disposition selon les termes de la Licence Creative Commons Attribution - Pas d'Utilisation Commerciale - Pas de Modification 4.0 International. 


\title{
Analyse multi-niveaux de l'activité en situation de formation : proposition méthodologique pour une étude synchronique et diachronique
}

\author{
Marlène Manach ${ }^{1}$
}

\begin{abstract}
Résumé
Cette contribution propose de rendre compte d'une méthodologie de construction des données empiriques issue de l'articulation entre l'approche sociotechnique des environnements de formation (Albero, 2010a/b/c) et le cadre théorique et méthodologique du cours d'action (Theureau, 2006, 2015), dans le cadre d'une analyse de l'activité en situation de formation. Cette articulation a pour but de comprendre l'activité des formateurs et des apprenants dans une perspective diachronique et synchronique, en s'intéressant à l'influence de la dimension sociale, matérielle et symbolique de l'environnement de formation sur l'activité et ses transformations successives.
\end{abstract}

Dans le champ de la formation des adultes, la recherche « orientée activité » (Barbier \& Durand, 2003) vise à répondre à des enjeux à la fois professionnels, scientifiques et sociaux. Dans cette perspective, cette contribution présente une proposition de méthodologie de recherche à partir d'une articulation entre le cours d'action développé en ergonomie (Theureau, 2006, 2015) et l'approche sociotechnique des environnements de formation en sciences de l'éducation et de la formation (Albero, 2010a/b/c). Cette articulation concerne deux cadres théoriques complémentaires qui partagent une même acception de l'activité humaine, à savoir un couplage entre un sujet agissant et un environnement qui lui apporte des ressources et des contraintes humaines, matérielles et symboliques (Albero, Guérin \& Watteau, 2019). Cette étude s'inscrit dans le cadre de travaux menés par d'autres chercheurs qui expérimentent cette articulation pour tenter de structurer, en sciences de l'éducation et de la formation, un cadre théorique et méthodologique commun autour de l'analyse de l'activité (Albero \& Guérin, 2014 ; Watteau, 2017).

Cette articulation vise à effectuer une analyse multi-niveaux de l'activité en situation de formation : une analyse meso correspondant à l'environnement de formation en s'intéressant aux intentions de formation des formateurs-concepteurs et à la mise en œuvre de ces intentions ; une analyse micro correspondant à l'activité effective des apprenants. Une étude diachronique et une étude synchronique sont réalisées. L'étude diachronique est une étude longitudinale visant à décrire les transformations successives de l'activité des apprenants pendant et après la formation. L'étude synchronique analyse les jeux de tension entre intentions de formation et activité effective des apprenants dans des situations de formation précises. L'enjeu est d'observer à la fois la temporalité de l'apprentissage et l'ici et maintenant des situations de formation.

Le texte présente les éléments qui constituent l'articulation des deux cadres ainsi que l'intérêt d'une articulation en termes de méthodologie. II décrit ensuite les étapes de la construction des données sur le terrain d'une formation aux compétences sociales.

\section{Fondements et objectifs d'une articulation épistémologique et théorique}

Le cadre épistémologique et théorique mobilisé s'inscrit dans le programme de recherche en sciences de l'éducation (Albero \& Guérin, 2014) visant à articuler l'approche sociotechnique (Al-

\footnotetext{
${ }^{1}$ Docteure en sciences de l'éducation, Centre de recherche sur l'éducation, les apprentissages et la didactique (CREAD), Université de Bretagne Occidentale. Cette étude s'inscrit dans le cadre d'une recherche bénéficiant d'une aide de l'État gérée par l'Agence nationale de la recherche au titre du programme Investissements d'avenir portant la référence nANR-11-IDFI-0035.
} 
bero, 2010a/b/c) et le cadre théorique et méthodologique du cours d'action (Theureau, 2006, 2015). Analyser l'activité dans cette perspective vise à documenter et à comprendre l'activité et ses transformations ${ }^{2}$ selon une approche holiste (Watteau \& Guérin, 2017) pour aider à concevoir des environnements de formation dans le cadre d'un espace de collaboration entre chercheurs, professionnels et usagers.

Un des fondements de l'articulation entre l'approche sociotechnique et le cours d'action est l'entrée activité, à savoir une centration sur l'observation, l'analyse de l'activité de l'individu et le sens qu'il lui accorde (Albero \& Guérin, 2014 ; Barbier, 2013). La recherche par une entrée activité repose sur le postulat que l'activité est une unité insécable (sujet et environnement, action et pensée forment un tout) et qu'elle constitue un processus dans lequel et par lequel est impliqué un individu en interaction avec son environnement naturel et social qu'il transforme et qui le transforme. De plus, le cours d'action et l'approche sociotechnique s'intéressent au concept de configuration d'activité pour appréhender différentes échelles d'analyse. Dans cette contribution, la notion de configuration est employée selon l'acception de Brigitte Albero (2000), c'est-à-dire comme un ensemble d'éléments présents dans la situation et qui instrumentent l'activité.

L'objectif de l'articulation est de mettre en relation l'analyse de l'environnement de formation et la manière dont il influence l'activité des apprenants (et des formateurs), en prenant en compte les artefacts $^{3}$. Le primat est accordé au point de vue des apprenants et des formateurs pour rendre compte de l'activité humaine in situ. L'analyse de l'activité des formateurs et des apprenants est effectuée pour tenter de mettre en regard les intentions pédagogiques avec le vécu et les processus de transformation de l'activité des apprenants. L'enjeu est d'identifier des configurations favorisant les transformations des activités et des individus en lien avec des intentions éducatives. En d'autres termes, il s'agit de savoir ce qui, dans l'environnement (les autres apprenants, les formateurs, les artefacts), instrumente l'activité et forme une configuration permettant à la formation de " faire dispositif », c'est-à-dire relevant d'un agencement intentionnel et stratégique d'humains et d'artefacts finalisés par un but (Linard, 1989), évolutif dans le temps et adaptable aux circonstances (Albero, 2011).

L'intérêt d'articuler cours d'action et approche sociotechnique est de permettre une analyse multiniveaux de l'activité (Albero, Guérin \& Watteau, 2019). En effet, la mobilisation seule du cours d'action, par son analyse à une échelle micro, peut négliger les incidences du contexte (environnement, moment, dimension socio-historique, etc.) sur le déroulement de l'activité et la signification donnée par les acteurs. La mobilisation seule de l'approche sociotechnique, par son analyse à une échelle méso et macro, peut réduire la réalité de l'activité effective aux pratiques explicitées. L'articulation entre cours d'action et approche sociotechnique, par leur complémentarité, vise donc à étudier sur plusieurs terrains les rapports entre trois échelles : 1) l'échelle macrostructurelle qui concerne l'environnement global ; 2) l'échelle meso-structurelle qui concerne l'environnement de formation et 3) l'échelle micro-structurelle qui concerne l'activité individuelle et collective instrumentée de manière matérielle et/ou symbolique.

\section{Recueil des matériaux empiriques}

Le terrain étudié est une formation visant le développement des compétences sociales (l'Initiative d'excellence en formation innovante, IDEFI - TalentCampus). Deux sessions de formation de cinq jours chacune ont été observées. Quatre participants volontaires ont été suivis pendant la formation puis trois mois et six mois après la formation. II s'agit de Christine, 54 ans ; Dan, 25

\footnotetext{
${ }^{2}$ L'apprentissage se construit dans l'action et est un processus continu de transformations de l'activité qui se renouvelle et se développe (Durand, 2007).

${ }^{3}$ L'artefact est défini comme « toute interface qui permet la réalisation d'une action humaine » (Simonian, 2015). L'artefact peut être naturel, matériel (un outil) ou symbolique (un concept) et est défini par celui qui l'utilise. L'artefact (matériel et symbolique) est susceptible d'un usage. II est élaboré pour produire une classe d'effets et atteindre un but, dans les conditions prévues par les concepteurs (Rabardel, 1995). L'artefact, externe au sujet, devient instrument lorsqu'il est intériorisé par l'acteur et approprié par lui (détourné, actualisé, transformé) en fonction de son histoire et de ses expériences antérieures (Albero, 2010b).
} 
ans ; Alice, 47 ans et Elen, 41 ans $^{4}$. En parallèle, trois formateurs-concepteurs et les deux fondateurs de TalentCampus ont été interrogés. La collaboration entre le chercheur et les participants a été contractualisée sur les plans éthique et technique (Guérin, 2012).

Le recueil des matériaux empiriques vise à renseigner le rapport entre les participants et l'environnement de formation (les autres apprenants, les formateurs, les artefacts) mais aussi à renseigner les intentions et actions des formateurs, de leur point de vue. Ce recueil a consisté en des enregistrements de l'activité suivis d'entretiens d'auto-confrontation, de remise en situation ou d'entretiens semi-directifs.

- Enregistrements de l'activité

Pour recueillir des traces de leur activité, les participants ont été filmés en continu durant les cinq jours de la formation. Une caméra sur trépied a été installée dans la salle de formation, suffisamment éloignée des participants et des autres apprenants pour éviter d'interférer dans leur activité. En parallèle, des notes ethnographiques ont été prises pour décrire les actions des apprenants et des formateurs, décrire la situation de formation ou noter des questions. Lorsque la captation vidéo n'était pas possible, ce qui a été le cas lors d'exercices en extérieur ou lorsque les participants n'étaient pas dans la même pièce, soit le seul son des micros installés sur les participants a été enregistré, soit des photos de l'activité ont été prises. Les trois types d'enregistrement de l'activité des participants (vidéo, son, photo) ont constitué les traces sur lesquelles chercheur et participant se sont appuyés pour les entretiens qui ont suivi et qui ont également été enregistrés.

\section{- Entretiens menés auprès des participants}

- Au cours de la formation (jours 1 à 5) : entretiens d'auto-confrontation et de remise en situation

À l'issue de chaque journée de formation, un entretien d'auto-confrontation a été mené auprès de chaque participant. Ce type d'entretien mobilise le cadre théorique et méthodologique du cours d'action (Theureau, 2010). II consiste en la confrontation des participants aux traces vidéo de leur activité, dans un temps différé (en l'occurrence, à l'issue de chaque journée de formation, du jour 1 au jour 5), pour qu'ils expriment la signification de leurs actions et de leurs communications ayant eu cours dans les interactions avec leur environnement (les autres apprenants, les formateurs, les artefacts). Lorsque l'activité n'avait pas pu être filmée, des entretiens de remise en situation par les traces de l'activité (photo ou enregistrement sonore) ont remplacé les entretiens d'auto-confrontation.

Selon la description de l'activité humaine de Jacques Theureau (2006), l'activité est vécue, c'està-dire qu'elle donne lieu à une expérience. Cette expérience est nommée conscience préréflexive et correspond au vécu accessible, à l'effet de surface du rapport entre l'acteur et son environnement. L'entretien d'auto-confrontation avait pour objectif l'expression de la conscience préréflexive des participants, c'est-à-dire de les encourager à montrer, raconter, mimer ou commenter ce qui avait été signifiant pour eux dans la situation. Pour cela, des questions leur ont été posées concernant leurs préoccupations, leurs focalisations, leurs actions ou leurs ressentis. À titre d'exemple: Qu'est-ce que tu ressens quand il te dit ça ? Là, qu'est-ce que tu fais ? Qu'estce qui fait que tu y vas à ce moment-là? Qu'est-ce qui attire ton attention ? ${ }^{5}$

\footnotetext{
${ }^{4}$ Des pseudonymes ont été utilisés pour préserver l'anonymat des participants.

${ }^{5}$ Lors de la phase de contractualisation avec les participants (explicitation du protocole de recherche) avant le début de la formation, le tutoiement a été choisi aussi bien par le chercheur que par les participants.
} 
- Après la formation (trois mois et six mois) : entretiens de remise en situation

Le recueil de matériaux visait à documenter l'objet théorique " cours de vie relatif à un projet " 6 (Theureau, 2015) dans le cadre de l'activité des participants. Des entretiens ont donc été menés trois mois puis six mois après la formation. Ces entretiens avaient pour objectif d'identifier dans quelle mesure les transformations qui avaient eu lieu pendant la formation s'actualisaient dans la vie quotidienne des participants. Ces entretiens se rapprochaient en partie des entretiens de remise en situation de second niveau décrits par Theureau (2010), dans le sens où il s'agissait de repartir des préoccupations, des actions et des transformations décrites pendant la formation, lors des entretiens d'auto-confrontation et de remise en situation afin de les commenter trois mois et six mois plus tard, lors d'un entretien semi-directif. L'objectif était, ensuite, de comparer les préoccupations, transformations, etc. pour observer les développements potentiels avant et après la formation.

\section{- $\quad$ Autres participants}

Quatorze autres participants volontaires ont été invités à décrire les moments signifiants de leur activité à un instant $t$, entre le jour 1 et le jour 5 lors d'entretiens semi-directifs. L'objectif de ces entretiens était de diversifier les données et de compléter les propos recueillis lors des entretiens menés avec les quatre participants suivis tout au long de la formation. II a été convenu avec ces quatorze participants que l'entretien serait fondé sur la question: quels ont été les moments marquants de ta formation? Les questions qui ont suivi s'appuyaient sur la même trame que les entretiens menés avec les quatre principaux participants.

\section{- Entretiens menés auprès des formateurs-concepteurs et des fondateurs}

Des entretiens semi-directifs ont été menés auprès des formateurs-concepteurs et des fondateurs de TalentCampus, à l'issue d'une journée de formation ou après la formation. L'objectif était de documenter les différentes logiques d'orientation de conduite de leur action, principalement les intentions de formation et la mise en œuvre de ces intentions à destination des apprenants pendant la formation. Pour cela, l'approche sociotechnique avec l'approche trilogique d'Albero (2010c, 2011) a été mobilisée en posant des questions se rapportant aux trois dimensions correspondant à trois aspects de l'activité des acteurs de la formation : l'idéel (idées, principes et objectifs), le fonctionnel de référence (définition des contenus, organisation des tâches et des modes d'action) et le vécu (appréhension et compréhension des situations) que nous illustrons avec les exemples suivants:

- l'idéel : quel est l'objectif de formation de ce module ? Quelle était ton intention en proposant cet exercice?

- le fonctionnel de référence : comment s'organise le débriefing en groupe ? Comment tu as préparé cette séance ? À quoi servent les outils proposés (cartes de jeux, sabliers, gommettes, etc.) ?

- le vécu : quel était ton état d'esprit en commençant la séance ? Quelque chose de particulier a-t-il attiré ton attention au cours de la séance?

Ces questions visent à renseigner les logiques d'orientation de l'action des formateursconcepteurs : la logique épistémo-axiologique (en lien avec l'idéel, les intentions de formation, les concepts, les modèles), la logique instrumentale (en lien avec le fonctionnel de référence, la mise en œuvre des actions de formation) et la logique existentielle (en lien avec le vécu au cours de l'action de formation). L'objectif est ensuite d'établir un lien entre ces logiques propres à l'action du formateur avec l'analyse de l'activité effective des apprenants pour identifier ce qui

\footnotetext{
${ }^{6}$ L'étude du cours de vie relatif à un projet (ou à une pratique) vise des activités humaines à long terme dans le cadre de ce projet. L'objet théorique « cours de vie » est défini par le fait que l'activité est montrable, racontable, commentable et donne lieu à une conscience préréflexive mais aussi qu'il existe un postulat de cohérence entre les épisodes disjoints d'un même projet (ou d'une même pratique) à travers le temps, malgré leur entrecoupement par des épisodes relatifs à d'autres projets.
} 
facilite ou empêche l'apprentissage dans la relation entre l'intention de formation, la mise en œuvre de l'action de formation et les intentions de l'apprenant (Albero, Guérin \& Watteau, 2019).

\section{Construction des données empiriques : études diachronique et synchronique}

La construction des données issues des matériaux empiriques résulte d'une construction en deux axes: une étude diachronique et une étude synchronique, pour tenter de comprendre l'activité humaine dans la complexité de son organisation temporelle (Theureau, 2006). Les deux études se sont appuyées sur des démarches méthodologiques distinctes mais complémentaires. L'étude diachronique s'appuie sur le cadre sémiologique du cours d'action (Theureau, 2006) pour documenter l'objet théorique " cours de vie relatif à un projet » (Theureau, 2015). L'objectif est de comprendre les transformations successives de l'activité des participants dans leur singularité. L'étude synchronique s'appuie sur les principes de la théorie ancrée (Glaser \& Strauss, 1967/2010) et a pour objectif d'analyser les données dans une démarche itérative, pour identifier ce qui structure l'activité des participants lors d'un épisode de formation.

L'articulation du cours d'action et de l'approche sociotechnique, dans sa dimension méthodologique, vise à construire des données dans une perspective holiste (Albero, Guérin \& Watteau, 2019) pour tenter de comprendre la complexité de l'activité individuelle et collective dans des environnements de formation instrumentés par des artefacts. Ainsi, l'étude diachronique se centre sur les processus de transformation de l'activité. L'étude synchronique vise à repérer ce qui, potentiellement, "fait dispositif » (Albero, 2011). Les données construites dans les deux études proviennent des mêmes matériaux (recueillis sur deux sessions de formation et trois mois puis six mois après chaque session de formation). Sur le terrain de la formation aux compétences sociales, cet ensemble de concepts et de méthodes est mobilisé au service de la compréhension des formes d'interactions entre des individus apprenants et leur environnement physique et social (les autres apprenants, les formateurs, les artefacts) et leurs influences sur le rapport à soi et aux autres.

\section{- Étude diachronique : cours de vie des participants}

L'étude diachronique propose d'analyser l'activité des participants en décrivant et analysant le cours de vie relatif au projet : transformer leur rapport aux autres. L'objectif est de rendre compte des transformations successives de leur activité dans leur rapport dynamique avec l'environnement physique et/ou social.

Le visionnage des entretiens d'auto-confrontation a permis de mettre en relation les données de verbalisation avec les données d'observation (films de l'activité, enregistrements sonores et photos) et de sélectionner des " données noyau » (Haué, 2003 ; Serres, 2006), c'est-à-dire les données les plus riches, correspondant aux épisodes les plus commentés par les participants et qui permettent une analyse qualitative fine. Le visionnage des vidéos a également permis de repérer des données relatives à un même projet pour les quatre participants, à savoir : transformer leur rapport aux autres.

\section{- Analyse sémiologique par le signe hexadique}

Pour comprendre la construction de l'expérience de chaque participant, les données noyau ont été analysées à un grain fin (analyse locale), en s'appuyant sur le cadre sémiologique de Theureau (2006). Cette analyse a permis de reconstruire l'organisation et la signification de chaque cours d'expérience (l'enchaînement des cours d'expérience correspondant au cours de vie) à partir des données de verbalisation relatives à la compréhension du vécu lors des entretiens d'auto-confrontation (Guérin, 2012). 
Dans le cadre sémiologique de Theureau, lorsqu'un acteur est invité à décrire un moment de son activité, il découpe le cours de cette activité en unités significatives pour lui. Le cours d'expérience de l'acteur est constitué par l'enchaînement de ces unités. Décrire et analyser le cours d'expérience consiste ensuite à rattacher ces unités à un ensemble de signes appelé «signe hexadique » qui correspond aux six composantes de l'expérience : l'engagement $(E)$, l'actualité potentielle $(A)$, le référentiel $(S)$; le représentamen $(R)$, l'unité significative élémentaire (USE) ; l'interprétant (I) ${ }^{7}$. Un signe hexadique décrit la conscience préréflexive de l'acteur, qui est une compréhension partielle par lui-même de son activité à un instant $t$, comme le passage d'un état de préparation de cet acteur (E-A-S) à un autre état ( $\left.E^{\prime}-A^{\prime}-S^{\prime}\right)$ (Sève et al., 2012). Dans le cadre de ce travail, la reconstruction des cours d'expérience avait pour but de repérer les préoccupations des participants et leur évolution en fonction du représentamen, c'est-à-dire en fonction de ce qui faisait signe pour eux. À titre d'exemple, le tableau ci-après présente un extrait de l'analyse sémiologique de l'activité d'une participante.

Tableau 1 - Extrait de l'analyse sémiologique de l'activité de Christine

\section{Séquence 1 - jour 1}

\section{Unités significatives élémentaires (USE)}

1. Se sent mal à l'aise dans le groupe.

2. Observe Valérie à de nombreuses reprises.

3. Se met en retrait vis-à-vis de Valérie.

4. Décide de relire les consignes sur sa tablette.

\section{Représentamen (R)}

1. Les membres du groupe ne se présentent pas.

2. L'attitude de Valérie (directive).

\section{État de préparation}

Engagement $(e R)$ : participer à un travail de groupe organisé.

Actualité potentielle $(a R)$ : s'attend à ce que les membres du groupe se présentent. Référentiel (sR) :

1. À l'habitude d'une certaine organisation dans le collectif de travail.

2. À besoin d'écoute dans le travail collectif.

3. Se met en retrait face aux personnes ayant une « forte personnalité ».

\section{Interprétant}

Émergence d'un type : l'investissement dans l'activité collective dépend du confort éprouvé avec les membres du groupe.

\section{Modification de l'état de préparation}

Engagement (eR') : avoir un rôle dans le groupe/s'investir dans le travail de groupe. Actualité potentielle (aR') :

1. Anticipe le fait de devoir produire (lecture des consignes sur la tablette).

2. S'attend à avoir des difficultés à interagir avec Valérie.

Référentiel ( $\left.s R^{\prime}\right)$ : a besoin d'être en confort avec le groupe.

La succession des analyses sémiologiques avait pour objectif de reconstruire le cours de vie de chaque participant relatif au projet : transformer son rapport aux autres.

\footnotetext{
${ }^{7}$ La documentation des six composantes de l'expérience a pour objectif de mettre en évidence : 1) l'intention de l'acteur dans la situation (engagement - E) ; 2) ce que l'acteur anticipe dans l'évolution de la situation (actualité potentielle - A) ; 3) les éléments de connaissance et d'expérience mobilisés par l'acteur dans la situation (référentiel - S) ; 4) ce qui fait signe (ce qui est pertinent pour l'acteur) dans la situation (représentamen - R) ; 5) ses actions, ses émotions, ses interprétations, ses communications (unités significatives élémentaires - USE) ; 6) les constructions de nouvelles connaissances (types) par l'acteur (interprétant - I)
} 


\section{- Synthèse de la reconstruction de l'expérience des participants}

Suite à l'analyse sémiologique (analyse locale) de l'activité des participants, une analyse globale a été effectuée. Les préoccupations ont été listées et regroupées pour observer leur évolution entre le jour 1 et le jour 5 de la formation.

La reconstruction du cours de vie des quatre participants a permis d'identifier des régularités dans les rapports entre les acteurs et leur environnement, notamment en ce qui concerne les préoccupations et leur évolution entre le jour 1 et le jour 5 de la formation. Quatre préoccupations types ont ainsi été identifiées, dans l'ordre chronologique de leur émergence : 1) s'investir dans la formation, respecter les consignes ; 2) appartenir au groupe, s'intégrer au groupe, avoir un rôle dans le groupe ; 3 ) interagir avec les autres de manière efficace (exprimer ses émotions, ses besoins, ses envies) ; 4) s'accepter, s'assumer (par l'acceptation de son rôle dans le groupe).

Les données issues des entretiens semi-directifs menés trois mois et six mois après la formation ont été transcrites en verbatim puis organisées en catégories (selon les mêmes principes que dans l'étude synchronique décrite au point suivant). Ce choix se justifie par le fait que seules des données de verbalisation ont été collectées ; il n'était donc pas possible de les associer à des données d'observation essentielles pour l'analyse locale.

Les verbalisations des participants à propos de leur activité après la formation ont été étiquetées puis catégorisées à partir des transcriptions verbatim des entretiens. Les modifications repérées dans l'activité des participants après la formation relevaient du rapport aux autres (en particulier dans les situations professionnelles) mais également : du rapport à soi (acceptation de soi, estime de soi) et de l'appropriation des artefacts introduits pendant la formation.

Bien que les cours de vie des quatre participants soient relatifs au projet de transformer leur rapport aux autres, ils sont décrits dans leur singularité dans la partie consacrée aux résultats. L'objectif est de mettre en évidence la singularité de l'expérience au sein d'un collectif d'apprenants ayant des préoccupations similaires.

\section{- Étude synchronique : analyse des cours d'action sur un épisode de formation}

L'étude synchronique consiste en une étude des différents cours d'action sur une situation ou épisode de formation. II s'agit de décrire et analyser les situations de formation (entre le premier et le cinquième jour) identifiées comme signifiantes du point de vue des participants (les quatre principaux participants et les quatorze autres apprenants interrogés) dans leur activité et de les mettre en regard avec les intentions formulées par les formateurs-concepteurs pour identifier ce qui instrumente les apprentissages et ce qui « fait dispositif ». L'entrée par les formés étant privilégiée, le vécu des formateurs-concepteurs n'est pas pris en compte. L'intérêt est de saisir l'influence de l'environnement de formation, sous-tendu par les intentions des formateursconcepteurs, sur l'organisation de l'activité des formés et de déceler les configurations (Albero, 2000) qui favorisent l'apprentissage.

\section{- Catégorisation de l'activité des participants}

Suivant les principes de la théorie ancrée (Glaser \& Strauss, 1967/2010), après avoir transcrit tous les entretiens menés avec les participants, les données verbatim ont été étiquetées. II s'agissait de tenter de nommer, qualifier, résumer les propos du verbatim phrase par phrase ou pour l'ensemble d'un paragraphe, en restant le plus fidèle possible à ce verbatim sans pour autant le répéter. Ces étiquettes ont été regroupées en catégories qui correspondaient à des régularités dans l'activité des participants et qui ont été construites sous la forme d'une problématisation progressive. 
À titre d'exemple, voici un extrait de la catégorisation faite à partir des données verbatim d'une participante. La première étape était la construction et la formulation des étiquettes par le chercheur à partir des transcriptions de la totalité des entretiens.

Tableau 2 - Exemple d'étiquetage des entretiens

Données verbatim extraites des entretiens

Extrait $n^{\circ} 1$ : Quand on est allés dans le parc parler à des inconnus, pour moi c'était difficile. Et d'arriver à se lancer, c'est quelque chose qui m'a rassurée dans ma capacité à faire face.

Extrait $n^{\circ} 2$ : Il a fallu que je reparte d'encore plus loin en termes de dépassement de soi. Ça m'a montré une force, celle d'arriver à me remonter toute seule. Ça m'a demandé un gros effort. Ça veut dire qu'en situation contrainte, j'arrive à le faire.

Extrait $n{ }^{\circ} 3$ : J'ai découvert un truc de moi, c'est que j'arrive bien à travailler sous pression.

\section{Étiquette}

La réalisation d'une tâche qu'elle considère difficile lui fait prendre conscience de sa force potentielle pour agir.

La deuxième étape a consisté pour le chercheur à regrouper les étiquettes en catégories de manière à signaler un phénomène, une idée plus large, pour un seul participant.

Tableau 3 - Exemple de construction de catégorie à partir des étiquettes

$\begin{array}{ll}\text { Étiquettes } & \text { Catégorie } \\ \text { À l'habitude de travailler en collectif. } & \begin{array}{l}\text { Rapport aux autres : } \\ \text { l'engagement individuel dans le } \\ \text { collectif semble orienté par le } \\ \text { beso sent d'appartenance, le be- }\end{array} \\ \text { À des attentes vis-à-vis du collectif. } & \begin{array}{l}\text { soin d'autonomie et le besoin } \\ \text { de sécurité. }\end{array} \\ \text { A besoin d'indépendance par rapport au collectif. } & \text { La position de dirigeant modifie } \\ \text { A aussi besoin d'appartenir au collectif. } & \text { les relations au collectif. } \\ \text { Agit après avoir été rassurée par les autres. } & \\ \text { Doit se préparer à un travail de coordination. } & \\ \text { Rejette la posture de « diriger » tout en espérant l'assumer dans un futur } & \\ \text { proche. } & \\ \text { N'est pas encore totalement à l'aise avec la prise de leadership. } & \end{array}$

II s'agissait ensuite de vérifier la validité des catégories en les confrontant aux données des autres participants. La comparaison entre les étiquettes des autres participants avait pour but de mettre en évidence les éléments constituant les catégories qui leur étaient communes. Les catégories ont ainsi pu être développées dans leur problématisation et certaines catégories ont été regroupées.

Lorsque les catégories se rapportaient à des composantes de l'activité-signe telles que des préoccupations, des constructions de connaissances ou ce qui était signifiant dans l'environnement, le lien a été fait entre la théorie ancrée et la théorie sémiologique du cours d'action, considérant qu'une préoccupation, par exemple, pouvait constituer une catégorie construite de manière inductive et itérative ${ }^{8}$. Par exemple, les étiquettes "veut avoir plus confiance en elle ", "cherche des signes de reconnaissance de soi par les autres ", " comprend que donner son opinion n'est pas être autoritaire ", extraites des verbatim de trois participants différents, ne sont pas seule-

${ }^{8}$ Cette articulation s'inspire des travaux de Jacques Saury \& Cécile Rossard (2009). 
ment relatives au rapport à soi ; elles concernent plus précisément une préoccupation ou une construction de connaissance relatives au rapport à soi.

Les catégories ont été considérées comme valides lorsqu'elles arrivaient à " saturation », c'està-dire lorsque les catégories étaient documentées par les données de tous les participants et suffisamment documentées pour qu'aucune autre étiquette ne vienne remettre en question ces catégories, "comme l'ajout de sel ne modifierait plus la salinité d'une solution saturée " (Paillé, 1994).

Huit catégories ont ainsi été élaborées, regroupant les étiquettes des quatre participants suivis pendant et après la formation et correspondant à ce qui structure leur activité :

1) le rapport à soi (préoccupations et construction de connaissances) ;

2) le rapport aux autres (préoccupations et construction de connaissances) ;

3) la perturbation des habitudes d'activité 9 par les éléments de l'environnement de formation (artefacts et collectif) ;

4) l'influence des émotions sur l'activité individuelle dans le collectif ;

5) l'influence de l'activité collective sur le rapport à soi ;

6) la transformation de l'image du fonctionnement collectif et de l'activité au sein du collectif ;

7) la transformation de la relation entre l'individuel et le collectif ;

8) le développement professionnel (après la formation).

\section{- Catégorisation des intentions de formation et des tâches}

En parallèle, il s'agissait d'identifier les tâches dans lesquelles l'activité des participants s'est déployée. II était donc nécessaire de connaître les objectifs et les consignes des exercices (traduisant les intentions des formateurs et leurs prescriptions) auxquelles ils se sont confrontés.

À partir des entretiens semi-directifs menés auprès des formateurs-concepteurs et des fondateurs et des documents pédagogiques, les intentions de formation ont été listées puis catégorisées de manière à déterminer des intentions macro. Six catégories ont ainsi été déterminées et correspondaient aux intentions de formation suivantes :

1) créer les conditions pour communiquer avec les autres de manière efficace ;

2) créer les conditions pour apprendre à se découvrir ;

3) créer les conditions pour sensibiliser à la dynamique de groupe (rôles, places dans un groupe, fonctionnement du groupe, collaboration/coopération) ;

4) créer les conditions pour apprendre à se dévoiler et à se valoriser ;

5) créer les conditions pour encourager la réflexion ;

6) mettre à disposition les outils pour agir en situation de collectif.

L'objectif était ensuite de décrire les tâches inhérentes à ces intentions et prescrites par les formateurs. Les tâches ont ensuite été regroupées par similitudes, lorsque cela était possible. Six catégories de tâches ont ainsi été déterminées:

1) se mettre en scène au sein d'un projet collectif ;

2) réfléchir sur soi ;

3) partager son point de vue après l'action (débriefing en groupe restreint) ;

4) prendre la parole devant les autres ;

5) réaliser un projet en groupe ;

6) découvrir une notion/une théorie présentée par les formateurs (débriefing en grand groupe).

Les tâches qui n'ont pas pu être regroupées ont été listées sous l'appellation « autres tâches ».

\footnotetext{
${ }^{9}$ Les habitudes d'activité (Barbier, 2017) sont des actions stabilisées provisoirement et mises en œuvre dans des situations récurrentes. Les habitudes sont des manières d'agir qui se construisent dans l'activité et se transforment avec cette activité.
} 
Enfin, un inventaire des matériaux disponibles par participant a été réalisé. Ces matériaux concernent les traces de l'activité et la verbalisation de l'activité par les participants pour chaque tâche.

Tableau 4 - Extraits des matériaux disponibles pour l'étude synchronique

Matériaux disponibles par participants

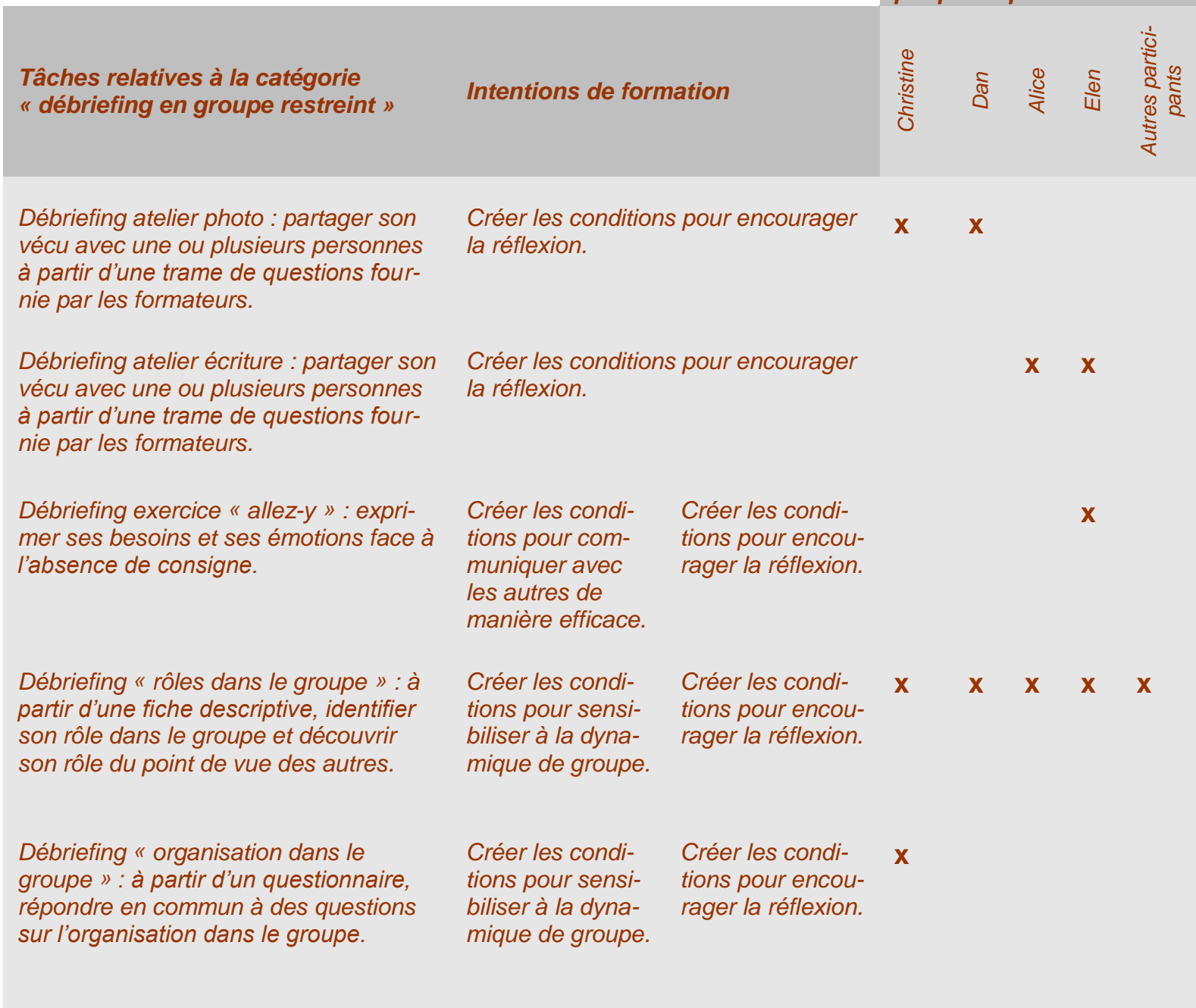

À l'issue de la construction des données pour l'étude synchronique, cinq situations ont été choisies pour être analysées, dont le débriefing "rôles dans le groupe " présenté dans le tableau cidessus. Cette situation a été choisie, car elle était commentée par la majorité des participants et récurrente dans la formation (un débriefing était organisé à l'issue de chaque exercice pour encourager la réflexion).

Les étapes suivantes de ce travail (résultats et discussion) ont pour objectif de décrire et analyser les cinq situations (intentions, tâches prescrites, action effective des apprenants) et de mettre en regard les logiques instrumentale et axiologique des formateurs et des fondateurs avec le vécu des apprenants, afin de mettre en évidence les convergences et les jeux de tension entre les différents acteurs de la formation (Albero, 2011 ; Archieri \& Jaouen, 2017). 


\section{Conclusion}

Analyser les données empiriques dans une perspective diachronique et synchronique vise à tenter d'analyser l'activité dans sa complexité, à la fois générale et singulière, générique et spécifique (Albero \& Guérin, 2014). L'articulation de l'approche sociotechnique et du cours d'action se fonde sur des postulats communs: l'activité doit être appréhendée comme un ensemble, où l'action et la situation forment un tout indissociable. Cette perspective aborde l'activité humaine comme étant autonome, incarnée, située et vécue.

L'enjeu de cette articulation est :

1) d'observer à la fois l'« ici et maintenant " des situations et la temporalité de l'apprentissage ; 2) d'avoir accès aux processus de construction de sens du sujet et les mettre en relation avec l'activité des formateurs ;

3) de comprendre les coordinations entre les activités individuelles et collectives ;

4) de s'intéresser à l'appropriation effective des artefacts par les apprenants ;

5) d'articuler l'analyse des niveaux inférieurs de l'activité (échelle micro-structurelle) avec celle des niveaux supérieurs (échelles meso et macro-structurelles) (Watteau, 2017).

L'articulation entre cours d'action et approche sociotechnique est donc une expérimentation visant à faire de la notion d'activité un cadre organisateur (Linard, 2001) pour la recherche et la formation (Albero \& Guérin, 2014). De manière générale, ce travail s'inscrit dans une finalité praxéologique (Albero \& Brassac, 2013), c'est-à-dire dans une finalité utilitaire pour le terrain d'étude (I'IDEFI TalentCampus) et d'autres environnements de formation.

\section{Références}

ALBERO Brigitte

- (2011), «Une approche trilogique des dispositifs de formation : pourquoi "les choses ne fonctionnent-elles jamais comme prévu ?" » dans Christine Gaux \& Isabelle Vinatier, Outils pour la formation, l'éducation et la prévention, En ligne http://cren.univ-nantes.fr

- (2010a), «Penser le rapport entre formation et objets techniques » dans Gilles Leclercq \& Renata Varga, Dispositifs de formation et environnements numériques : enjeux pédagogiques et contraintes informatiques, Paris, Hermès/Lavoisier.

- (2010b), « Une approche sociotechnique des environnements de formation : rationalités, modèles et principes d'action », Éducation et didactique, vol.1, n4, p.7-24.

- (2010c), «La formation en tant que dispositif : du terme au concept » dans Bernadette Charlier \& France Henri (dir.), La technologie de l'éducation: recherches, pratiques et perspectives, Paris, Presses universitaires de France.

- (2000), L'autoformation en contexte institutionnel: du paradigme de l'instruction au paradigme de l'autonomie, Paris, L'Harmattan.

ALBERO Brigitte, GUÉRIN Jérôme \& WATTEAU Benjamin (2019), « Comprendre la relation entre influences de l'environnement et activité : questionnements théoriques et enjeux praxéologiques », Savoirs, n49, p.103-124.

ALBERO Brigitte \& GUÉRIN Jérôme (2014), "L'intérêt pour l' " activité » en sciences de l'éducation : vers une épistémologie fédératrice ? », Transformations : recherches en éducation et formation des adultes, n¹1, p.11-45.

ALBERO Brigitte \& BRASSAC Christian (2013), « Une approche praxéologique de la connaissance dans le domaine de la formation. Éléments pour un cadre théorique ", Revue française de pédagogie, n¹84, p.105-120.

ARCHIERI Catherine \& JAOUEN Pierre-Yves (2017, juin), “ Un dispositif innovant à l'épreuve de l'approche sociotechnique: une collaboration aux finalités évolutives », communication présentée au $4^{\mathrm{e}}$ colloque international de didactique professionnelle, Entre pressions institutionnelles: quelles analyses de l'activité en situation de travail en didactique professionnelle?, Villeneuve-d'Ascq (France).

BARBIER Jean-Marie (2017), Vocabulaire d'analyse des activités. Penser les conceptualisations ordinaires, Paris, Presses universitaires de France ( $2^{\mathrm{e}}$ édition). 
BARBIER Jean-Marie (2013), «Un nouvel enjeu pour la recherche en formation : entrer par l'activité », Savoirs, vol.33, $n^{\circ} 3$, p.9-22.

BARBIER Jean-Marie \& DURAND Marc (2003), « L'activité : un objet intégrateur pour les sciences sociales ? ", Recherche \& Formation, n42, p.99-117.

DURAND Marc (2007), « Situations de l'action, dispositions à agir et trajectoires d'activité chez des enseignants débutants et des formateurs de terrain ", Formation et pratiques d'enseignement en question, n6, p.83-98.

GLASER Barney \& STRAUSS Anselm (2010), La découverte de la théorie ancrée. Stratégies pour la recherche qualitative, Paris, Armand Colin.

GUÉRIN Jérôme (2012), Activité collective et apprentissage: de l'ergonomie à l'écologie des situations de formation, Paris, L'Harmattan.

HAUÉ Jean-Baptiste (2003), «Étude de l'activité du quotidien de gestion d'énergie dans une finalité de conception », communication présentée aux Journées d'Act'Ing, Saint-Pierre de Quiberon (France).

LINARD Monique (2001), "Concevoir des environnements pour apprendre: l'activité humaine, cadre organisateur de l'interactivité technique », Sciences et techniques éducatives, vol.8, n`3-4, p.211-238.

LINARD Monique (1989/1996, revu et augmenté), Des machines et des hommes : apprendre avec les nouvelles technologies, Paris, L'Harmattan

PAILLÉ Pierre (1994), “L'analyse par théorisation ancrée », Cahiers de recherche sociologique, n²3, p.147181.

RABARDEL Pierre (1995), Les hommes et les technologies. Approche cognitive des instruments contemporains, Paris, Armand Colin.

SAURY Jacques \& ROSSARD Cécile (2009), "Les préoccupations des élèves durant des tâches d'apprentissage coopératives et compétitives en badminton: une étude de cas ", Revue des sciences de l'éducation, vol.35, n³, p.195-216.

SERRES Guillaume (2006), Analyse de la construction de l'expérience professionnelle au gré des diverses situations de formation initiale des enseignants du second degré, Thèse de doctorat, Université Blaise Pascal Clermont-Ferrand II.

SĖVE Carole, THEUREAU Jacques, SAURY Jacques \& HARADJI Yvon (2012), " Drôles d'endroits pour une rencontre : STAPS, ergonomie et cours d'action » dans Matthieu Quidu, Les sciences du sport en mouvement. Innovations et traditions théoriques en STAPS, Paris, L'Harmattan.

SIMONIAN Stéphane (2015), L’affordance socio-culturelle: une approche éco-anthropocentrée des objets techniques, Habilitation à diriger des recherches, Université Rennes 2.

THEUREAU Jacques (2015), Le cours d'action : l'enaction et l'expérience, Toulouse, Octarès.

THEUREAU Jacques (2006), Le cours d'action : méthode développée, Toulouse, Octarès.

THEUREAU Jacques (2010), «Les entretiens d'autoconfrontation et de remise en situation par les traces matérielles et le programme de recherche "cours d'action" ", Revue d'anthropologie des connaissances, vol.2, $n^{\circ} 4$, p.287-322.

WATTEAU Benjamin (2017), Approche écologique de l'activité de création en formation : le cas de l'atelier de projet en architecture, Thèse de doctorat, Université Rennes 2.

WATTEAU Benjamin \& GUÉRIN Jérôme (2017, novembre), «An ecological approach of human activity in training environment: from targeted learning to actual transformation », communication présentée au colloque ESREA, Exploring learning contexts: implications for access, learning careers and identities, Rennes (France). 